\title{
Minimally altering a critical kinase for low-phytate maize
}

\begin{abstract}
Alla Singh*, Chikkappa Karjagi \& Sujay Rakshit
Nutritional security is of vital importance for combating malnutrition and catering to increasing energy demands. Phytic acid is considered an anti-nutrient, which sequesters important metal ions, limiting their bioavailability. The lpa mutants of maize contain reduced phytate, thus increase its nutritive value. But low phytate is accompanied by negative pleiotropic effects. This article discusses the importance of lpa2 gene amongst available options, for precise DNA editing to simultaneously improve nutrition and avoid pleiotropic effects.
\end{abstract}

One major objective of millennium development goals (MDGs) is the eradication of extreme poverty and hunger. A call was made in the form of sustainable development goals (SDGs) to achieve zero hunger by 2030 . Consistent and sustained efforts are being made across the globe to achieve food security, mainly in developing countries of Asia, Africa and Latin America. More effort is needed if the SDGs are to be met timely ${ }^{1}$. Maize is an important staple crop as food and feed in many parts of the world. In developing countries, malnutrition is a public health problem. The rapidly expanding population on one hand, and the capacity of middle income groups to pay for higher nutrition on the other, demands production of high-quality foods. Thus of late, more emphasis is being given on the quality of food for humans and feed for animals. Improvement of nutritional value of food crops, either by enhancing the nutrient levels through bio-fortification or by reducing the anti-nutritional factors like phytates would substantially increase their biological value, either due to improved digestibility and/or bioavailability ${ }^{2,3}$. In case of maize, phytic acid is one such anti-nutritional factor which sequesters important metal ions like $\mathrm{Mg}^{++}, \mathrm{Fe}^{++}, \mathrm{Zn}^{++}, \mathrm{K}^{+}$etc., thus reducing the bioavailability of these micronutrients ${ }^{4}$.

Phytic acid is the hexa-phosphorylated form of myo-inositol. Chemically, phytic acid is the myo-inositol $(1,2,3,4,5,6)$-hexakisphosphoric acid. The major portion of phytic acid is stored in seeds, mainly in the form of mixed salts of mineral cations, referred to as phytate. In maize, more than $80 \%$ of phytic acid is stored in seed germ, it acts as the reservoir of phosphorous and inositol ${ }^{5}$. Phytic acid is synthesized by two pathways, namely lipid- dependent (operates in all plant organs) and lipid- independent (predominant in seeds) ${ }^{6}$. Phytic acid is packed into storage vacoules and other organelles. Due to sequestration of metal ions by phytic acid, the bio-availability of these ions from food gets limited in humans and other monogastric animals. Reduction of phytic acid content is hence, an important breeding goal in crop plants.

Many natural mutants with reduced phytate in different crops have been identified. However, reduction in phytic acid content has been associated with negative pleiotropic effects like decreased germination percentage and weight of seeds. Inositol phosphates play an important role in several cellular functions, including lipid metabolism. Impairment of the metabolic functions, otherwise carried out by inositol phosphates, in the phytic acid-related natural mutants could be a reason for their negative pleiotropic effects 6 . In this context, the challenge is to reduce the phytic acid levels without introducing substantial negative pleiotropic effects. Therefore, it is important to choose the best gene target(s) to accomplish the desired outcome, which is reduced phytic acid content with minimal off-target effects. Raboy has summarized the pathway of phytic acid synthesis, starting from glucose-6-phosphate and different targets have been indicated for reducing phytate ${ }^{7}$. Various natural mutants like soybean LR33; maize lpa3-1, lpa2-2, lpa1-1; Arabidopsis atipk1, atipk2 etc. can be potentially targeted. The mutants in various crops, the responsible loci, amount of phytate reduction and other phenotypic effects associated with the mutant have been summarized in Sparvoli and Cominelli 6 . Three classes of maize mutants with reduced phytic acid content, referred to as low phytic acid or lpa, are known. Raboy et al. have described the origin and phenotype of seed phytic acid mutants lpa 1 and lpa 2 in maize ${ }^{8}$. Shi et al. characterized lpa 3 mutants of maize ${ }^{9}$. In terms of phytic acid biochemistry, lpa 3 is the most upstream and encodes a myo-inositol kinase, which phosphorylates myo-inositol to inositol monophosphates. lpa2 encodes Inositol Phosphate Kinase 1 (IPK1), which phosphorylates inositol-5-phosphate ( $\left.\mathrm{IP}_{5}\right)$ to inositol-6-phosphate $\left(\mathrm{IP}_{6}\right)$ or phytic acid. lpa1 is the most downstream of all and encodes an ABC (ATP binding cassette) - transporter protein that packages phytic acid into various vacuoles. In many natural mutants, the expression of the encoded protein is switched off. Proteins 
are usually involved in interactions with many other proteins. Null expression or silencing of a protein results in disruption of protein-protein interactions and signaling or downstream metabolic networks associated with such protein-protein interactions. Disruption of protein-protein interaction networks comprises a potential reason for the diverse negative pleiotropic effects linked with natural or engineered mutants. Raboy has enlisted four potential target areas, which include (a) inhibition of the synthesis of myo-inositol and inositol-3-phosphates, (b) inhibition of synthesis of inositol-6-phosphate (phytic acid), (c) inhibition of transport and storage of phytic acid in the cell, and (d) expression of phytase encoding transgenes ${ }^{10}$. Given the public aversion to genetically modified foods in certain countries, the last approach is limited in practical value, hence the first three may be considered for a broader applicability. Genome editing gives an opportunity to modify specific gene(s) to achieve desirable effect ${ }^{11}$. In this regard, it is essential to prioritize target gene(s), for achieving precise engineering of physiological processes with minimal negative pleiotropic effects associated with changes in a particular gene sequence(s). One possible strategy to avoid off-target effects is to mutate a protein through gene editing to result in a variant that has reduced or null enzymatic activity. The edited protein would thus block the concerned metabolic reaction. However, the protein would still be fully expressing to fulfill its function of interacting with other proteins. Targeting the upstream reactions of a metabolic pathway would result in a snow-ball effect, influencing all the other downstream processes. Hence, inhibition of myo-inositol and inositol-3-phosphates would disrupt not only the inositol metabolism, but all other allied processes associated with it. Inositol phosphates are involved in a range of cellular functions like membrane transport, cell division, cytodifferentiation, regulatory role in signal transduction (lipid signalling) etc ${ }^{12}$. On the other hand, blocking the transport of phytic acid in cellular compartments has been described as a viable strategy. Shi et al. silenced embryo-specific expression of an ABC transporter protein that transports phytic acid from cytoplasm to vacuole ${ }^{13}$. The authors report that while the null lpa1 mutant has attenuated seed germination, the lpa1-1 point mutant displays normal germination percentage. However, the seed weight of point mutant is lesser than normal maize. Landoni et al. have demonstrated changes in physical properties of $l$ pa 1 maize, including modifications in density, starch properties, fiber content etc., which has implications for the practical utility of $l p a 1$ maize $^{14}$. The available evidence shows that phytic acid can be packaged into cell organelles via multiple transporter proteins of the ABC Multiple Drug Resistance associated Protein (MRP) type. In Arabidopsis, the phytic acid is known to be packaged in at least three compartments: protein storage vacuole of embryo, endoplasmic reticulum and vacuolar compartments of chalazal endosperm ${ }^{15}$. The storage in last two compartments is transient. Due to multiple transport compartments, silencing of one transporter protein in one compartment may not be a viable strategy. Further, the accumulation of phytic acid in cytoplasm has the potential to lead to toxicity, as phytic acid at defined concentrations has been demonstrated to possess cytotoxicity in human cell lines ${ }^{16}$. Either or combination of the above phenomenon may be responsible for the decreased seed weight of lpa1 mutants.

Being the most downstream enzyme in the metabolic pathway of phytic acid formation, Inositol Phosphate Kinase 1 or IPK1 is a potential gene target for achieving reduction in phytate content. IPK1 catalyzes the phosphorylation of inositol phosphates to higher phosphorylated forms like $\mathrm{IP}_{6}$ (Phytic acid). It is also involved in protein-protein interactions with other proteins. IPK1 can thus be mutated, such that the phosphorylation of different inositol phosphates does not take place, but the protein remains intact for protein-protein interactions to take place. Figure 1 shows the current understanding of phtyic acid formation and transport in cell. Studies have shown that apart from inositol phosphates, inositol pyrophosphates also play an important role in cellular metabolism $^{17,18}$. Phytic acid is further converted to higher phosphorylated forms by Inositol hexakisphosphate kinase (IP6K) enzyme. This leads to the formation of inositol pyrophosphates $\mathrm{IP}_{7}\left(\mathrm{PP}-\mathrm{IP}_{5}\right)$ and $\mathrm{IP}_{8}\left(2\left(\mathrm{PP}-\mathrm{IP}_{4}\right)^{19}\right.$. The available literature suggests that the function of $\mathrm{IP}_{7}$ and $\mathrm{IP}_{8}$ can be carried out by inositol pyrophosphates $\mathrm{PP}_{-\mathrm{IP}_{4}}$ and $2(\mathrm{PP}) \mathrm{IP}_{3}{ }^{19}$. Saiardi et al. have shown that a null mutant of IPK1 (ipk1 $\Delta$ ) in yeast results in accumulation of pyrophosphates $\mathrm{PP}_{-} \mathrm{IP}_{4}$ and $2(\mathrm{PP}) \mathrm{IP}_{3}$ besides $\mathrm{IP}_{5}$, yet resembles wild-type cells in morphology ${ }^{19}$. Abnormal vesicular morphology in case of null mutations of other inositol phosphate kinases is attributed to the loss of inositol pyrophosphates, which does not happen in case of $i p k 1 \Delta$, thus explaining its similar morphology to wild type cells. Hence, loss of phytic acid would not impair the function mediated by $\mathrm{IP}_{7}$ and $\mathrm{IP}_{8}$, since $\mathrm{PP}_{\mathrm{IP}}$ and $2(\mathrm{PP})$ $\mathrm{IP}_{3}$ impart functional redundancy by complementation [Fig. 1(f,g)]. In view of the above and multiple transport routes of phytic acid along with protein-protein interactions of IPK1, it appears to be the critical kinase which can be targeted without introducing multiple negative plieotropic effects, as observed with other mutants. The IPK1 enzyme can be minimally altered by disrupting its catalytically active site, so that the protein is fully expressing, but unable to convert IP 5 to phytic acid. The desired minimal alteration in IPK1 would only affect phytic acid formation, leaving the upstream and downstream process, as well as the protein-protein interactions intact.

In order to evaluate the prospects of minimally altering Zea mays IPK1, a computational model of the protein was made using PSI-BLAST (Position-Specific Iterative Basic Local Alignment Search Tool) based structure prediction $^{21}$. The structure was refined by side-chain repacking ${ }^{22}$. The refined structure contains $13 \alpha$-helices and $15 \beta$-sheets, with $92.4 \%$ residues in Rama-favoured region and no poor rotamers. The Class (C), Architecture (A), Topology (T), superfamily (H) analysis ${ }^{23,24}$ of the modeled Zea mays IPK1 structure showed it to contain the structure typically found in inositol phosphate kinases. The substrate inositol pentkisphosphate $\left(\mathrm{IP}_{5}\right)$ was docked to the IPK1 model using a rigid docking algorithm ${ }^{26,27}$, which was further refined ${ }^{28,29}$. Both $\mathrm{IP}_{5}$ and cofactor Adenosine triphosphate (ATP) bind in a cleft formed by four $\beta$-sheets from residues 195-202, 205$212,278-286,292-299$ and two $\alpha$-helices 260-273 \& 310-326. Analysis of the docked structure using PDBsum web server ${ }^{30,31}$ showed Alanine 5, Histidine 205, Threonine 207 and Cysteine 208 to be closely interacting with $\mathrm{IP}_{5}$ (Fig. 2A). The proteins that interact with IPK1 via protein-protein interactions include acid phosphatase, inositol-pentakisphosphate 2-kinase, inositol polyphosphate multikinase, succinate-CoA ligase, inositol 3-kinase, $\mathrm{ABC}$ MRP4 transporter and a metal ion binding protein (Fig. 2B). Mutation of the IPK1 protein at key amino acids that result in destabilization of the protein in its active site or hinder interactions with substrate or cofactor will result in a protein, functional for protein-protein interactions but non-functional for phytic acid formation. 


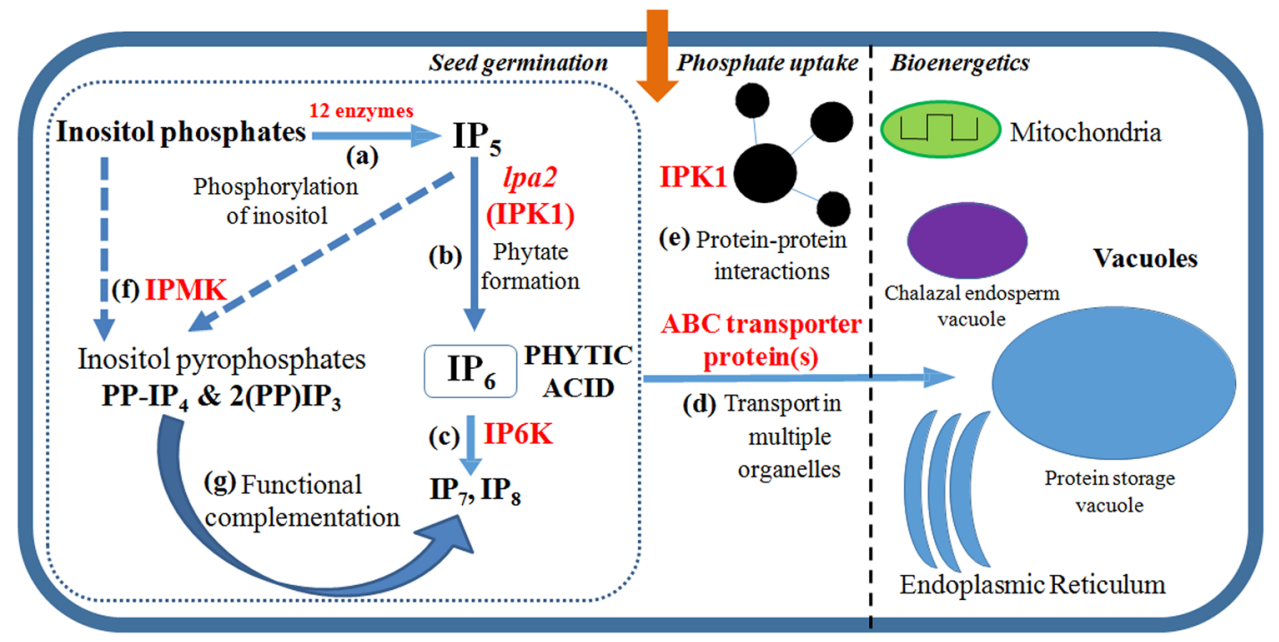

Figure 1. A model of key components of inositol phosphate pathway involving IPK1. (a) myo-Inositol is converted to inositol phosphates, including $\mathrm{IP}_{5}$ via 12 enzymes. (b) IPK1 then phosphorylates $\mathrm{IP}_{5}$ to $\mathrm{IP}_{6}$ or phytic acid. (c) IP6K phosphorylates phytic acid to higher forms like $\mathrm{IP}_{7}$ and $\mathrm{IP}_{8}$. (d) Phytic acid is transported to different organelles via $\mathrm{ABC}$ transporter proteins into storage vacuole, chalazal endosperm vacuole and endoplasmic reticulum. (e) IPK1 is involved in protein-protein interactions with other proteins, which are involved in seed germination, phosphate uptake and bioenergetics. (f) Inositol phosphate multi-kinase ( IPMK) converts $\mathrm{IP}_{5}$ and other inositol phosphates to inositol pyrophosphates $\mathrm{PP}_{-} \mathrm{IP}_{4}$ and $2(\mathrm{PP})-\mathrm{IP}_{3}$. (g) Inositol pyrophosphates $\mathrm{PP}_{\mathrm{IP}}$ and $2(\mathrm{PP})-\mathrm{IP}_{3}$ are capable of mediating functions carried out by $\mathrm{IP}_{7}$ and $\mathrm{IP}_{8}$, which would not be formed in the event of absence of $\mathrm{IP}_{6}$. IPK1 itself, rather than a single transporter protein or an upstream enzyme, appears to be the most promising target for low-phytate maize.

A.

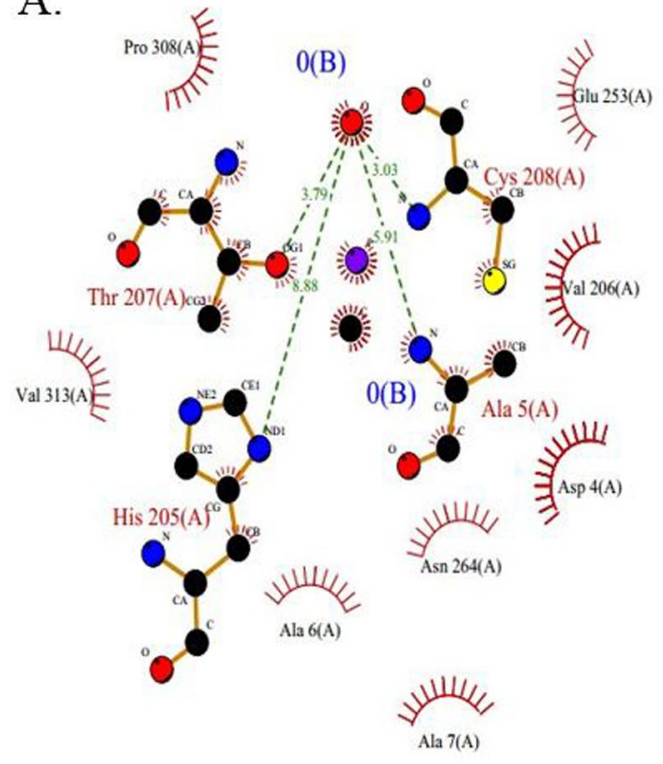

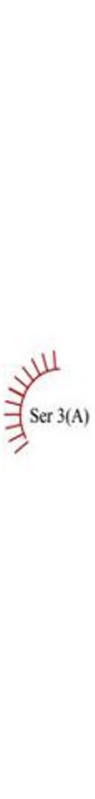

B.

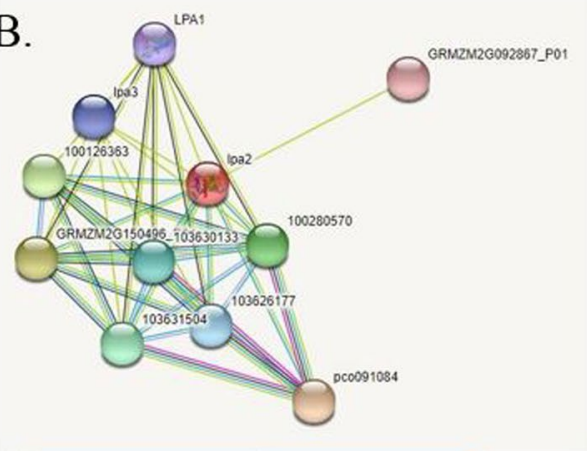

\begin{tabular}{|c|c|}
\hline C. Mutation & Predieted $\triangle \Delta G$ \\
\hline H205Y & 1.483 \\
\hline H205W & 1.049 \\
\hline H205Y & 1.042 \\
\hline H205L & 0.853 \\
\hline H205V & 0.739 \\
\hline H205C & 0.563 \\
\hline
\end{tabular}

Figure 2. Computational model of IPK1 with its substrate, its interacting proteins and identification of key mutations for catalysis inhibition. (A) Residue of IPK1 that interact with substrate IP $_{5}$. (B) Protein-protein interactions of IPK1 indicating associated proteins, revealed by databases and textmining. (C) Mutations that destabilize protein at 205 position and have the potential to hinder catalytic activity.

In the present case, Histidine 205 is implicated to be important for protein stability. Various mutations at His205 position have the potential to destabilize the protein, thereby hindering its function of phosphorylation (Fig. 2C). Similarly, alanine mutants of other interacting residues have the potential to inhibit phosphorylation by IPK1.

Cowieson et al. have postulated a term 'phytate-free nutrition' to emphasize the importance of reducing the content of phytic acid in feed ${ }^{20}$. The authors mention the fact that prominence is being given to formulation of phytate-free diets rather than accommodating phytase enzyme in the diet, as majority of phytic acid is digested 
in ruminants, but recalcitrant phytic acid does not get digested. If phytic acid content is reduced in the first place, mineral bioavailability would be enhanced. At the same time, efforts must be directed to minimize any possibilities for off-target effects of phytate reduction on plants. The above study is a step towards prioritization of target genes for dephytinization of maize to enhance its nutritional value.

\section{Methods}

Generation of protein model. The DNA sequence of lpa2 encoding for inositol phosphate kinase 1 was taken from National Center of Biotechnology Information. It was modelled through Bioserf (available on PSIPRED webserver) ${ }^{21}$. The obtained model was refined through GalaxyRefine program ${ }^{22}$. The modelled structure was analysed for class, architecture, topology, superfamily through CATH/Gene3Dv4.2 program²3,24.

Docking of substrate and cofactor with protein. The substrate inositol pentakisphosphate $\left(\mathrm{IP}_{5}\right)$ and cofactor Adenosine triphosphate (ATP) were downloaded from ZINC database ${ }^{25}$. The substrate and cofactor were docked on to the modelled protein using Patchdock algorithm ${ }^{26,27}$ and refined using Firedock webserver ${ }^{28,29}$. The interacting residues were identified using PDBsum ${ }^{30,31}$.

Protein interactions and prediction of stability at key residues. The protein-protein interactions of IPK1 were obtained using STRING database ${ }^{32}$. The stability of protein with mutation at key residues was determined using Site Directed Mutator program ${ }^{33}$.

Received: 21 November 2019; Accepted: 16 March 2020;

Published online: 14 April 2020

\section{References}

1. Tracking progress on the SDGs. Nat. Sustain. 1, 377 (2018).

2. Bouis, H. E. \& Saltzman, A. Improving nutrition through biofortification: A review of evidence from HarvestPlus, 2003 through 2016. Glob. Food Sec. 12, 49-58 (2017).

3. Garcia-Oliveira, A. L., Chander, S., Ortiz, R., Menkir, A. \& Gedil, M. Genetic Basis and Breeding Perspectives of Grain Iron and Zinc Enrichment in Cereals. Front. Plant Sci. 9, 937 (2018).

4. Gupta, R. K., Gangoliya, S. S. \& Singh, N. K. Reduction of phytic acid and enhancement of bioavailable micronutrients in food grains. J. Food Sci. Technol. 52, 676-684 (2015).

5. Raboy, V. Seed Phosphorus and the Development of Low-Phytate Crops in Inositol Phosphates. Linking Agriculture and the Environment (eds. Turner, B. L., Richardson, A. E. \& Mullaney, E.J.) 111-132 (CABI, 2006).

6. Sparvoli, F. \& Cominelli, E. S. Biofortification and Phytic Acid Reduction: A Conflict of Interest for the Plant?. Plants (Basel, Switzerland) 4(4), 728-755 (2015).

7. Raboy, V. The ABCs of low-phytate crops. Nat. Biotechnol. 25, 874-875 (2007).

8. Raboy, V. et al. Origin and Seed Phenotype of Maize low phytic acid 1-1 and low phytic acid 2-1. Plant Physiol. 124(1), 355-368 (2000).

9. Shi, J., Wang, H., Hazebroek, J., Ertl, D. S. \& Harp, T. The maize low-phytic acid 3 encodes a myo-inositol kinase that plays a role in phytic acid biosynthesis in developing seeds. Plant J. 42(5), 708-719 (2005).

10. Raboy, V. Approaches and challenges to engineering seed phytate and total phosphorus. Plant Sci. 177(4), 281-296 (2009).

11. Perry, M. E., Valdes, K. M., Wilder, E., Austin, C. P. \& Brooks, P. J. Genome editing to 're-write' wrongs. Nat. Rev. Drug Discov. 17(10), 689-690 (2018).

12. Livermore, T. M., Azevedo, C., Kolozsvari, B., Wilson, M. S. \& Saiardi, A. Phosphate, inositol and polyphosphates. Biochem. Soc. Trans. 44(1), 253-259 (2016).

13. Shi, J. et al. Embryo-specific silencing of a transporter reduces phytic acid content of maize and soybean seeds. Nat. Biotechnol. 25(8), 930-937 (2007).

14. Landoni, M. et al. Low Phytic Acid 1 Mutation in Maize Modifies Density, Starch Properties, Cations, and Fiber Contents in the Seed. J. Agric. Food Chem. 61(19), 4622-4630 (2013).

15. Otegui, M. S., Capp, R. \& Staehelin, L. A. Developing seeds of Arabidopsis store different minerals in two types of vacuoles and in the endoplasmic reticulum. Plant Cell 14(6), 1311-1327 (2002).

16. de Lima, E. M., Kanunfre, C. C., de Andrade, L. F., Granato, D. \& Rosso, N. D. Cytotoxic effect of inositol hexaphosphate and its $\mathrm{Ni}(\mathrm{II})$ complex on human acute leukemia Jurkat T cells. Toxicol. In Vitro 29(8), 2081-2088 (2015).

17. Williams, S. P., Gillaspy, G. E. \& Perera, I. Y. Biosynthesis and possible functions of inositol pyrophosphates in plants. Front. Plant Sci. 6, 67 (2015).

18. Shears, S. B. Inositol pyrophosphates: why so many phosphates? Adv. Biol. Regul. 57, 203-216 (2015).

19. Saiardi, A., Sciambi, C., McCaffery, J. M., Wendland, B. \& Snyder, S. H. Inositol pyrophosphates regulate endocytic trafficking. Proc. Natl. Acad. Sci. 99, 14206-14211 (2002).

20. Cowieson, A. J., Ruckebusch, J. P., Knap, I., Guggenbuhl, P. \& Fru-Nji, F. Phytate-free nutrition: A new paradigm in monogastric animal production. Animal Feed Sci. and Technol. 222, 180-189 (2016).

21. Buchan, D. W., Minneci, F., Nugent, T. C., Bryson, K. \& Jones, D. T. Scalable web services for the PSIPRED Protein Analysis Workbench. Nucl. Acids Res. 41, W349-W357 (2013).

22. Ko, J., Park, H., Heo, L. \& Seok, C. GalaxyWEB server for protein structure prediction and refinement. Nucl. Acids Res. 40, W294-W297 (2012).

23. Dawson, N. L. et al. CATH: an expanded resource to predict protein function through structure and sequence. Nucl. Acids Res. 45, D289-D295 (2017).

24. Lewis, T. E. et al. Gene3D: Extensive prediction of globular domains in proteins. Nucl. Acids Res 46, D435-D439 (2018).

25. Sterling, T. \& Irwin, J. J. ZINC 15-Ligand Discovery for Everyone. J. Chem. Inf. Model 55(11), 2324-2337 (2015).

26. Duhovny D. N. R. \& Wolfson, H. J. Efficient Unbound Docking of Rigid Molecules in Lecture Notes in Computer Science 2452 (eds. Guig'o, R. \& Gusfield, D.) 185-200 (WABI 2002).

27. Schneidman-Duhovny, D., Inbar, Y., Nussinov, R. \& Wolfson, H. J. PatchDock and SymmDock: servers for rigid and symmetric docking. Nucl. Acids Res. 33, W363-367 (2005).

28. Andrusier, N., Nussinov, R. \& Wolfson, H. J. FireDock: fast interaction refinement in molecular docking. Proteins 69(1), 139-159 (2007).

29. Mashiach, E., Schneidman-Duhovny, D., Andrusier, N., Nussinov, R. \& Wolfson, H. J. FireDock: a web server for fast interaction refinement in molecular docking. Nucl. Acids Res. 36, W229-W232 (2008). 
30. Laskowski, R. A. et al. PDBsum: a Web-based database of summaries and analyses of all PDB structures. Trends Biochem. Sci. 22(12), 488-490 (1997)

31. Laskowski, R. A., Jabłońska, J., Pravda, L., Vařeková, R. S. \& Thornton, J. M. PDBsum: Structural summaries of PDB entries. Protein Sci. 27(1), 129-134 (2018).

32. Szklarczyk, D. et al. STRING v11: protein-protein association networks with increased coverage, supporting functional discovery in genome-wide experimental datasets. Nucl. Acids Res. 47, D607-D613 (2019).

33. Worth, C. L., Preissner, R. \& Blundell, T. L. SDM--a server for predicting effects of mutations on protein stability and malfunction. Nucl. Acids Res. 39, W215-W222 (2011).

\section{Author contributions}

A.S. envisaged the concept and performed computational analysis. A.S., C.K. and S.R. compiled the model of phytic acid dynamics, based on current understanding. A.S., C.K. and S.R. wrote the manuscript.

\section{Competing interests}

The authors declare no competing interests.

\section{Additional information}

Correspondence and requests for materials should be addressed to A.S.

Reprints and permissions information is available at www.nature.com/reprints.

Publisher's note Springer Nature remains neutral with regard to jurisdictional claims in published maps and institutional affiliations.

(c) (i) Open Access This article is licensed under a Creative Commons Attribution 4.0 International License, which permits use, sharing, adaptation, distribution and reproduction in any medium or format, as long as you give appropriate credit to the original author(s) and the source, provide a link to the Creative Commons license, and indicate if changes were made. The images or other third party material in this article are included in the article's Creative Commons license, unless indicated otherwise in a credit line to the material. If material is not included in the article's Creative Commons license and your intended use is not permitted by statutory regulation or exceeds the permitted use, you will need to obtain permission directly from the copyright holder. To view a copy of this license, visit http://creativecommons.org/licenses/by/4.0/.

(C) The Author(s) 2020 